\title{
O PAPEL DA ENFERMAGEM NA REABILITAÇÃO FÍSICA
}

\author{
Maria do Socorro de Vasconcelos Campos ${ }^{1}$, Roberto Del Valhe Abi Rached ${ }^{2}$ \\ 1 Graduanda de Enfermagem. Universidade Nove de Julho - UNINOVE. São Paulo - Brasil - Email: São Paulo - \\ Brasil - email: regiane0255@gmail.com \\ 2 Doutor em Medicina; Faculdade de Medicina da USP -. São Paulo - Brasil email: robertoabira- \\ ched@yahoo.com.br
}

\section{RESUMO}

Objetivo: Demonstrar o papel do Enfermeiro na reabilitação física, levando em consideração todos os aspectos de educação e capacitação aos cuidadores e familiares antes da programação da alta hospitalar. Método: Trata-se de uma pesquisa de revisão literária, a fim de identificar as papel da enfermagem na reabilitação física. Utilizaram-se as bases de dados LILACS, SCIELO, no período de 2014 a 2018. Resultados: Demonstra a importância da assistência de enfermagem na reabilitação físico-motora devido o aumento significativo no número de pessoas com doenças crônico-degenerativas. Conclui-se que a atuação de enfermeiro é essencial no processo educativo de reabilitação com pacientes e seus familiares, para independência funcional, prevenção de complicações secundárias, adaptação as sequelas

Palavras chaves: Reabilitação, Enfermeiro, Enfermagem.

\begin{abstract}
Objective: To demonstrate the role of nurses in physical rehabilitation, taking into account all aspects of education and training of caregivers and family members prior to scheduling hospital discharge. Method: This is a literature review, in order to identify the role of nursing in physical rehabilitation. We used the LILACS and SCIELO databases from 2014 to 2015. Results: It demonstrates the importance of nursing care in physical and motor rehabilitation due to the significant increase in the number of people with chronic-degenerative diseases. It is concluded that nurses' performance is essential in the educational process of rehabilitation with patients and their relatives, for functional independence, prevention of secondary complications, adaptation to sequelae
\end{abstract}

Key words: rehabilitation, nurse, nursing

\section{INTRODUÇÃO}

No Brasil, a reabilitação começou a ser desenvolvida no início dos anos 50, devido à epidemia de poliomielite que acometeu o país. Na década de 1970, a Irmandade da Santa Casa de Misericórdia de São Paulo organizou em seu Hospital um Serviço de Reabilitação dedicado à 
prática do atendimento precoce da incapacidade e à prevenção da invalidez. A incapacidade deixou de ser compreendida como a consequência inevitável de doença ou trauma e passou a ser tratada profilaticamente por uma equipe multiprofissional em todas as unidades de internação.

As estatísticas sobre reabilitação são insuficientes, porém, segundo estimativas da Organização das Nações Unidas, o país apresenta, em média, 10\% de sua população com algum grau de incapacidade.

A demanda de pacientes com algum grau de incapacidade necessitando de cuidados em reabilitação vem aumentando ${ }^{1}$.

A imobilidade poderá ser entendida como um sério problema de saúde pública, uma vez que aqueles que dela sofrem apresentam ou podem vir a apresentar sérios danos na sua saúde, acarretando dificuldades que esta impede em grande medida a independência do doente na realização das suas atividades de vida diárias².

É importante que sejam traçados metas e objetivos que possam contribuir para a diminuição de fatores estressores, como envolve-lo como coautor de sua história ${ }^{2}$.

O enfermeiro habilitado em enfermagem de reabilitação avalia, cria e implementa programas de reabilitação que visam a melhoria da funcionalidade e minimizar as incapacidades. As suas intervenções são direcionadas para o paciente/família. A capacitação do paciente /família é preponderante na eficácia da reabilitação ${ }^{1}$.

A reabilitação envolve a utilização de técnicas e ações interdisciplinares, como o esforço conjunto de todos os profissionais e familiares, dentro e fora das instituições e que deve ter como objetivo comum a melhora e/ou a reabilitação das funções diminuídas ou perdidas para preservar a capacidade de viver de cada indivíduo ${ }^{3}$.

Um dos princípios que tem norteado a afirmação e reconhecimento da especialidade de enfermagem de reabilitação, está relacionado em elaborar, mapear os desafios em planos e projetos. A materialização destes desafios inicia-se na etapa formativa dos enfermeiros especialistas, fomentando a aquisição de competências e o espírito de renovar os mesmos para o exercício da profissão ${ }^{2}$.

O programa de reabilitação exige que o paciente/família aprenda a viver em um novo cenário de vida, com todas as modificações ocorridas, com maior incidência na função motora, sensitiva e cognitiva. A abordagem precoce da enfermagem de reabilitação é fundamental na melhora da funcionalidade e redução das limitações, através da adequação e das intervenções mediante à avaliação diagnóstica e envolvimento do paciente/família na implementação das mesmas. O processo de capacitação do paciente/família é importante para a reabilitação funcional e prevenção de incapacidades secundárias ${ }^{3}$. 
Entretanto, não há um sistema de classificação especificamente direcionado à assistência de enfermagem em reabilitação físico-motora, embora seja crescente o número de indivíduos a demandar esta especialidade de cuidado ${ }^{4}$.

O enfermeiro, como líder de equipe de Enfermagem, desenvolve diversas ações gerenciais. Dentre elas, podem-se citar: o cuidado e o acompanhamento do paciente por Intervenções de enfermagem ao paciente através do provimento de material e pessoal necessários para melhorar o processo de reabilitação e realizar a capacitação de equipe multidisciplinar relacionada às técnicas de manipulação, mobilidade e transferência dos pacientes. Além disso, supervisionam e impulsionam os pacientes, a cada dia, a realizarem suas atividades de autocuidado ${ }^{4}$.

A Enfermagem apresenta-se como campo de mudanças globais, promovendo estas, relacionadas à forma de fazer e entender o cuidado, assim como as demandas geradas pela mudança populacional e as implicações para cuidadores, familiares e profissionais. Soma-se as mudanças populacionais estruturais relacionadas à transição demográfica e epidemiológica, àquelas nos sistemas familiares e sociossanitários e, particularmente, nas formas de entender e fazer o cuidado 5 .

Mediante a pesquisa, tem se o propósito de revisar a literatura, discutir o papel do Enfermeiro no processo de reabilitação; no contexto educação: paciente, familiar, cuidador.

Identificar a contribuição do Enfermeiro junto a equipe multidisciplinar de reabilitação.

\section{OBJETIVO}

Analisar a prática em reabilitação por meio da enfermagem, aliada com a educação e cuidado ao paciente, o que contribuirá para uma maior eficácia e influência no processo e nos serviços de reabilitação.

\section{METODOLOGIA}

As bases de dados selecionadas para a realização da busca bibliográfica foram: Base de dados: Latin American and Caribean Health Science Literature Database (Lilacs), acessada pelo portal da Biblioteca Virtual de Saúde Os critérios de inclusão estabelecidos para os estudos foram: a) disponíveis nos idiomas português, inglês ou espanhol; b) completos que abordem cuidados de Enfermagem direcionados aos pacientes adultos e idosos com necessidade de cuidados na fase de reabilitação; c) que apresentem qualidade metodológica A busca bibliográfica ocorreu entre os anos de 2014 e 2018, com gravação da página de busca Após a busca bibliográfica, os estudos levantados inicialmente nas bases de dados, foram lidos para que o pesquisador aplicasse, em sua totalidade, os critérios de inclusão. Esta etapa ocorreu de 12 abril a 14 de junho de 2018 . 
Os critérios de inclusão estabelecidos para os estudos foram: a) disponíveis nos idiomas português, inglês ou espanhol; b) completos que abordem cuidados de Enfermagem direcionados aos pacientes adultos e idosos com acidente vascular cerebral na fase de reabilitação; c) que apresentem qualidade metodológica.

\section{DESENVOLVIMENTO}

Olhar para o aumento do número de pessoas com alterações da mobilidade constitui também um desafio para os enfermeiros de reabilitação, no sentido de que lhes exige que as suas intervenções tenham como objetivo minorar as limitações impostas pela situação clínica, dignificando deste modo esta etapa da vida. Alguns estudos têm demonstrado o aumento da prevalência das doenças crónicas degenerativas em pessoas com mais de 65 anos e que as alterações da mobilidade são o maior problema da pessoa que envelhece ${ }^{6}$ A reabilitação é um processo dinâmico, orientado para a saúde, que auxilia um indivíduo que está incapacitado para atingir seu maior nível possível de funcionamento físico, mental, espiritual, social e econômico. O processo de reabilitação ajuda a pessoa a atingir uma aceitável qualidade de vida com dignidade, autoestima e independência .

O trabalho de reabilitação da pessoa portadora de limitação física no Brasil começou a ganhar espaço, principalmente, a partir do XXIX Congresso Brasileiro de Enfermagem, que aconteceu por volta de 1980. A assistência de enfermagem na reabilitação tem como principais objetivos auxiliar o paciente a tornar- -se independente o máximo que puder dentro de suas condições, promover e incentivar o autocuidado através de orientações e treinamento de situações, preparar o deficiente físico para uma vida social e familiar com qualidade.

Os principais cuidados prestados pelos enfermeiros aos pacientes de Reabilitação, na visão dos próprios enfermeiros em um estudo realizado em um hospital público da cidade de São Paulo, são: prevenção de úlceras por pressão; realização de cateterismo vesical intermitente; estimulação à deambulação; avaliação da perfusão distal; orientações para curativos; utilização de colchão piramidal, coxins e meias elásticas; orientações ao paciente e família sobre atividades básicas da vida diária, entre outros(5)

o enfermeiro deve supervisionar e estimular exercícios; e realizar atividades de educação e aconselhamento para a redução de riscos. Também é papel do enfermeiro fornecer o conhecimento para uma vida mais saudável, apoio e encorajamento necessários ao paciente durante o processo de reabilitação.

Para os autores, o enfermeiro deve incentivar o paciente a sentar-se, engatinhar e andar, de acordo com a idade; implementar e instruir a família na realização de exercícios de extensão (para prevenir deformidades); incentivar o autocuidado, de acordo com a idade e capacidade; 
ajudar no controle da mandíbula durante a alimentação; utilizar objetos e figuras (para reforçar a fala e incentivar a compreensão); ensinar e utilizar métodos de comunicação não-verbal, quando necessário; elogiar a criança por suas realizações e quase realizações, como a execução parcial de uma tarefa; evitar críticas; orientar os pais sobre a importância de seu interesse e apoio no desenvolvimento do autocuidado de seus filhos.

Observa-se que a reabilitação físico-motora é um importante componente no processo saúdedoença e no cuidado psicossocial de pessoas com sequelas motoras e neurológicas. Isto ocorre devido ao aumento significativo no número de pessoas com doenças crônico-degenerativas e das que sobrevivem a lesões neurológicas, como acidente vascular encefálico, lesão medular, e trauma crânio-encefálico. ${ }^{3}$

Entretanto, não há um sistema de classificação especificamente direcionado à assistência de enfermagem em reabilitação físico-motora, embora seja crescente o número de indivíduos a demandar esta especialidade de cuidado.

Durante a consulta de enfermagem, além do levantamento relativo aos dados pessoais, de saúde e da família, inclui-se a "Avaliação das atividades da vida". Cada atividade deve ser avaliada em termos da função e ação, buscando a identificação de rotinas anteriores, execução atual e problemas atuais e em potencial do idoso. Após a análise dos dados, os problemas são identificados e escritos.

Para a elaboração do "Planejamento da assistência de enfermagem", devemos considerar os recursos disponíveis em relação ao ambiente físico, aos equipamentos como o uso de acessórios e ao recurso pessoal, compreendido como enfermagem, família, serviços de apoio, entre outros. O plano de enfermagem não é estático e portanto, requer uma revisão contínua e coleta de dados adicionais a partir da valiação inicial. Os objetivos a serem alcançados são traçados junto ao idoso, em termos do seu desempenho esperado após a intervenção de enfermagem.

\section{CONSIDERAÇÕES FINAIS}

Conclui-se que a atuação do enfermeiro na reabilitação física é ampla e que muitas ações podem ser desenvolvidas junto ao paciente, independente de qual comorbidade tenha atingido o paciente. Os cuidados de enfermagem de reabilitação compreendem um corpo de conhecimentos e procedimentos específicos, que permitem intervir na manutenção das capacidades funcionais desses doentes, prevenir complicações e manter ou recuperar a independência nas atividades de vida, através de técnicas específicas, proporcionando o direito à dignidade e à qualidade de vida. Estes são cuidados ativos apropriados à condição de saúde dos doentes e suas famílias. São ainda, cuidados globais e abrangem os aspetos físicos, psicológicos, emo- 
cionais e espirituais, envolvendo a reabilitação. A reabilitação física e educação a alguma adaptação ao corpo, é uma das faces da Enfermagem. A atuação do enfermeiro na equipe multidisciplinar está centrada no processo educativo com pacientes e seus familiares, tendo como finalidade a sua independência funcional, a prevenção de complicações secundárias, sua adaptação e da família à nova situação.

\section{REFERÊNCIAS}

1. VIDAL, AA; PADULA, Capelett, MP. A Enfermagem em reabilitação física como tema de Trabalhos de Conclusão de Curso (TCC) de Graduação em Enfermagem. Arquivos Médicos dos Hospitais e da Faculdade de Ciências Médicas da Santa Casa de São Paulo, v. 57, n. 3, p. 97-102, 2018.

2- Lima, AMN. A reabilitação e a independência funcional do doente sujeito a imobilidade: [dissertação] Viana do Castelo: Instituto do Castelo, Viana, Portugal; 2014

3- Freitas, C. Intervenção do enfermeiro especialista em enfermagem de reabilitação na prevenção de complicações da espasticidade que interferem na marcha decorrentes de lesão neurológica: [tese de doutorado] Santarém: Instituto Politécnico de Santarém, Santarém, Portugal;2015

4- Souza, DRP de et al. Terms of International Classification for Nursing Practice in motor and physical rehabilitation. Rev da Esc de Enferm da USP.2015; 49(2); 0209-0215

5- Cavalcante, ES et al. Homens vítimas de lesão medular em reabilitação: uma análise contextual. Rev de Enferm UFPE.2015; on line-ISSN: 1981-8963, v. 9, n. 10, p. 9601-9607.

6.Alves, ,MCF. Os Cuidados de Enfermagem de reabilitação na Mobilidade do Doente Paliativo não Oncológico: perspetiva do Enfermeiro de reabilitação. MS thesis. 2014. 